\title{
DETERMINAÇÃO DA CONCENTRAÇÃO DE PARTÍCULAS EM UMA SEÇÃO RISER PELA TÉCNICA DE VELOCIMENTRIA POR IMAGEM
}

\author{
M. C. G. ROSA, G. J. de CASTILHO \\ Universidade Estadual de Campinas, Faculdade de Engenharia Química, Departamento de \\ Engenharia de Processos \\ E-mail para contato: matheus.guerta@gmail.com \\ E-mail para contato: guilhermejc@feq.unicamp.br
}

RESUMO - A determinação da concentração de partículas e a sua velocidade são importantes para descrever fenômenos em uma série de experimentos. No riser, seção principal de um Leito Fluidizado Circulante (LFC), há dois tipos de regimes relativos à concentração de sólidos: o regime diluído e o regime denso. O primeiro ocorre a velocidades de gás elevadas, baixas concentrações de sólidos e baixas quedas de pressão por unidade de comprimento de linha de transporte, já o regime em fase densa caracteriza-se por baixas velocidades de gás, alta concentração de sólidos e altas quedas de pressão por unidade de comprimento. Como a concentração e a velocidade afetam o regime da seção riser de um LFC, acabam por afetar seu desempenho, portanto a análise destas é importante. E para realizar tais análises é necessária a técnica de velocimetria por imagem de particulado do tipo tomográfica (TomoPIV), uma expansão da técnica de velocimetria de particulado por imagem (PIV) capaz de avaliar a concentração por meio da distribuição de densidade de particulado no leito e os vetores de velocidades em um espaço 3D.

\section{INTRODUÇÃO}

Leitos fluidizados circulantes (LFC) são sistemas com diversas aplicações na indústria, abrangendo mistura, secagem, reações catalíticas e não-catalíticas, entre outras. Estes sistemas são compostos basicamente de riser, alimentador de partículas, downer, alimentador de ar. A seção principal de um sistema LFC é o reator riser, pois neste pode ocorrer a quebra de hidrocarbonetos pesados em compostos mais leves e, portanto, de maior valor agregado. Seu escoamento gás-sólido é classificado em dois tipos: denso e diluído. Sendo que no regime diluído encontram-se velocidades de gás elevadas, baixas concentrações de sólidos e baixas quedas de pressão por unidade de comprimento de linha de transporte, já o regime em fase densa caracteriza-se por baixas velocidades de gás, alta concentração de sólidos e altas quedas de pressão por unidade de comprimento (CASTILHO, 2011).

Como as variáveis velocidade e concentração afetam o tipo de regime do riser, acabam afetando o desempenho deste também. Portanto, analisá-las é importante e para realizá-las é necessário o uso da técnica de velocimetria por imagem de particulado (PIV). A técnica emprega partículas traçadoras que seguem fielmente o escoamento, para extrair a velocidade do fluído. As partículas espalham luz em uma lente fotográfica, localizada a $90^{\circ}$ da folha de luz, de modo que o seu plano do objeto em foco coincide com a fatia iluminada do 
fluido. As imagens são formadas em um detector de arranjo vídeo e são, em seguida, transferidas para um computador para análise automática (ADRIAN, 1991).

Porém, para conseguir obter os vetores velocidade e a distribuição de densidade de particulas em três dimensões, é preciso utilizar sua expansão, a técnica de velocimetria por imagem de particulado do tipo tomográfica ou Tomo-PIV, que através da intensidade de cada partícula estima a posição desta no espaço (AMARAL, 2013).

\section{MATERIAIS E MÉTODOS}

Para a determinação da concentração e dos vetores na velocidade, em uma seção riser do LFC, foi empregada a técnica de velocimetria por imagem de particulado do tipo tomográfica (Tomo-PIV). Para a realização da técnica foram necessários o software Davis 8.0, quatro câmeras CCD (explicar o que é CCD), dois lasers, uma placa de calibração (Type 22) e, então, seguiram-se as etapas descritas abaixo operando o leito com uma vazão de ar 81 $\mathrm{Nm}^{3} / \mathrm{h}$, inversor de freqüência a $45,15 \mathrm{~Hz}$.

Utilizando a equação obtida por Cresmasco pode ser calculado o a vazão mássica do LFC, onde $\mathrm{m}=0,0465 *$ (frequência do inversor) - 0,2967, resultando em 1,803 $\mathrm{g} / \mathrm{s}$ (CASTILHO, 2007).

\subsection{Calibração}

O processo de calibração é a primeira etapa e é o mais importante da técnica TomoPIV, pois é responsável por definir a qualidade dos dados que serão obtidos. Neste se escolhem o tipo de placa (no experimento, foi usado Type 22), a função de mapeamento (função para se localizar partículas no plano num espaço 3D) e a quantidade de câmeras para o experimento que,no caso Tomo-PIV, são necessárias 4 câmeras (LAVISION, 2010).

Espera-se que ao final desta, para experimentos em condições ideais, o erro seja aproximadamente de 0,1 pixel (LAVISION, 2010).

\subsection{Aquisição de imagem}

Realizada a calibração, a etapa seguinte é a aquisição de imagem. Nela, escolhe-se um tempo entre cada sequência de imagem (dt). Então, enquanto o laser1 dispara, cada câmera captura uma imagem do fluído em análise e num tempo dt após o laser2 dispara e cada câmera captura uma nova imagem. Essa obtenção de imagem é importante, pois é através dela que serão analisadas as variáveis velocidade e concentração.

\subsection{Pré-processamento de imagem}

Após a aquisição de imagem, há o pré-processamento de imagem. Ele consiste na aplicação de filtros para remoção e correção de brilhos ou de seções, melhoria do contraste. $\mathrm{O}$ objetivo desta etapa é ter uma imagem com uma qualidade melhor e, assim, obter melhores resultados (LAVISION, 2010).

\subsection{Autocalibração}


A autocalibração é um procedimento realizado em 5 etapas para corrigir a função de mapeamento e possíveis desalinhamentos das câmeras (AMARAL et al., 2012).

A primeira etapa é a detecção de posições 2D das partículas. Nesta, as partículas com intensidade maiores são selecionadas evitando partículas fantasmas - partículas com intensidade menores que não representam pontos reais, geradas por intersecção dos focos das câmeras (LAVISION, 2010).

Realizada a detecção de posições 2D das partículas, há a triangulação das posições de imagem 3D das partículas. Seu objetivo é corresponder todas partículas do plano 2D em posições 3D do espaço, verificando se em cada câmera há uma partícula correspondente ao redor das posições $\mathrm{X}=(\mathrm{X}, \mathrm{Y}, \mathrm{Z})$ (LAVISION, 2010).

Então, prosseguindo, a próxima etapa consiste na construção de um mapa de disparidades. Nesta etapa, o volume iluminado é divido em volumes menores (subvolumes).ç No subvolume são recolhidas todas as partículas 3D para o tempo inicial (t0) e o tempo dt após (t1). As disparidades são exibidas como um histograma, na forma de bolhas pequenas de Gauss 2D (WIENEKE, 2008).

Por último, com as informações obtidas na etapa anterior e utilizando um procedimento semelhante ao da correlação cruzada faz com que a sejam gerados vetores. Esse procedimento recebe o nome de extração de vetores de disparidade e após sua realização a função de mapeamento é corrigida. Além disso, a sequência de etapas da autocalibração pode ser repetida até que se obtenha próximos a 0,1 pixel (WIENEKE, 2008).

\subsection{Reconstrução tomográfica}

$\mathrm{Na}$ reconstrução tomográfica, ocorre a estimativa da partícula que se encontra no plano 2D para um espaço 3D usando a intensidade. Nesta etapa também se escolhe a função de reconstrução com base em dois critérios: tempo de processamento e qualidade dos resultados. Para esta a análise a técnica utilizada foi a Fast-MART.

\subsection{Distribuição da densidade de partícula 3D}

Antes de se obter os vetores de velocidade, a distribuição de densidade pode ser obtida. Este procedimento permite avaliar a concentração através de duas formas: por unidade de volume ou por unidade de volume de interrogação, sendo que na unidade de volume, o resultado gerado é dado por partículas $/ \mathrm{cm}^{3}$ e no por volume de interrogação é feito uma média das partículas por cada pequena subdivisão do volume.

\subsection{Correlação Cruzada}

Nesta última etapa, se obtém os vetores de velocidade. Ela consiste em selecionar pequenas regiões (volumes de interrogação) e determinar a diferença das posições no tempo t0 e t1 resultando num vetor de velocidade (PRASAD, 2000). 


\section{RESULTADOS}

Os métodos foram seguidos e primeiramente realizou-se a calibração, apresentando os erros para cada câmera exibidos na Tabela 1 .

Tabela 1: Erros de câmara após a realização da calibração

\begin{tabular}{|l|l|l|l|l|}
\hline & camera 1 & camera 2 & camera 3 & camera 4 \\
\hline Stdev of fit & 0.568 & 0.68 & 0.983 & 0.622 \\
\hline
\end{tabular}

Como o experimento foi realizado em condições não ideais (presença de variações causadas pelo material e luz ambiente), foi considerado um erro máximo de 1 pixel para cada câmera como razoável.

Após a calibração prosseguiu-se para a obtenção de imagens. Nela foi escolhido um tempo de $100 \mu$ s para que houvesse um deslocamento médio de 20 pixels das partículas e, desta forma, ser possível a realização da análise de concentração e dos vetores velocidades. E, então para cada câmera foi obtida uma imagem e elas foram pré-processadas resultando em imagens semelhante à Figura 1.

Figura 1: Imagem das partículas obtidas na etapa de aquisição de imagem

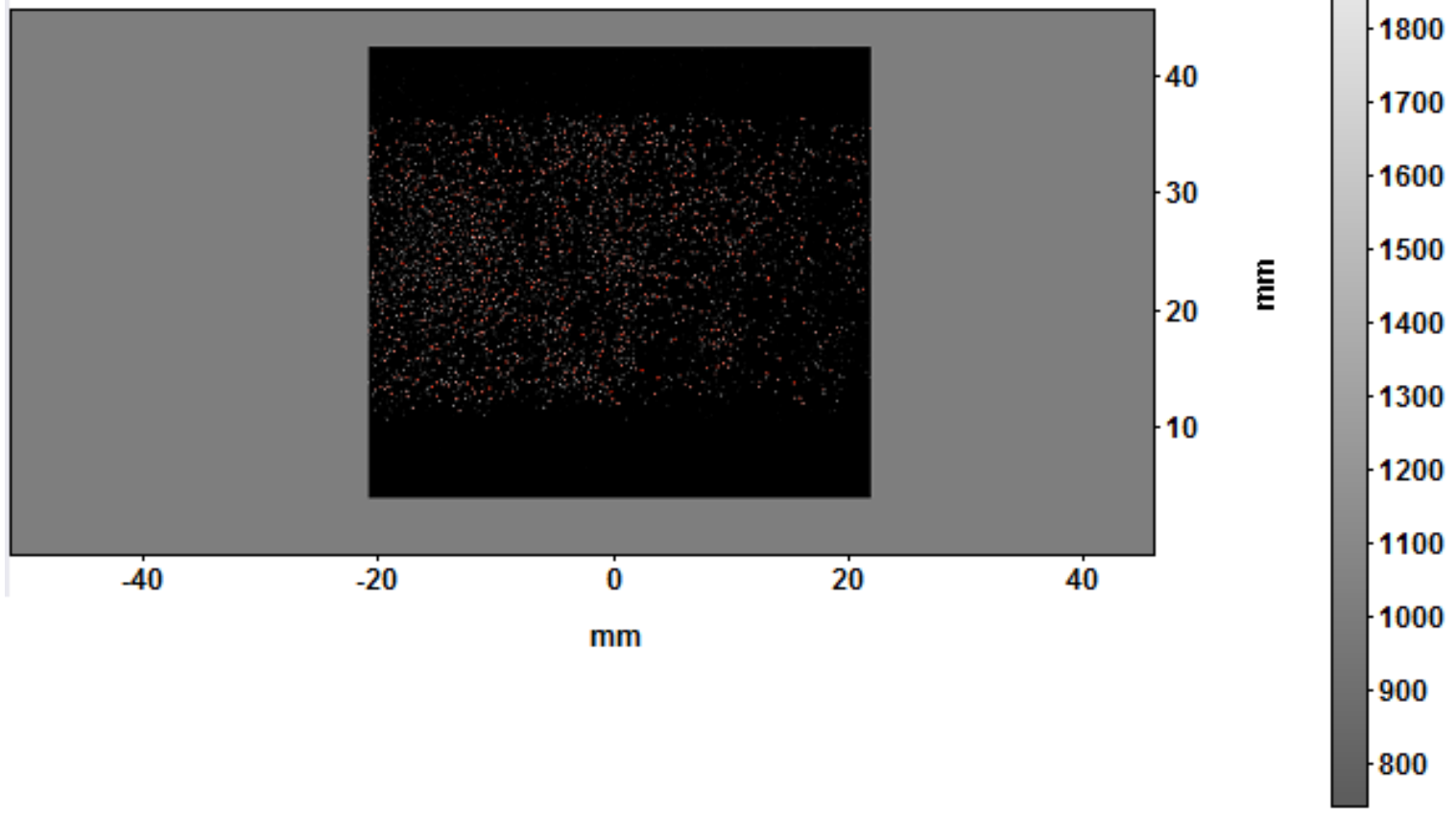

Na Figura 1 é possível verificar dois eixos. O eixo horizontal representa a posição $\mathrm{x}$ da figura no plano, o vertical representa a posição y. A escala de cores representa o valor de intensidade das partículas e este que será usado para localizar a partícula no espaço posteriormente. 
Antes da obtenção dos dados de velocidade, as imagens foram usadas para correção da função de mapeamento e um possível desalinhamento das câmeras. Pode-se então, reconstruir o volume e os dados da distribuição de concentração foram obtidos, resultando na Figura 2 para o tempo t0. Os eixos x e y representam a localização no plano xy, já o eixo z se refere a concentração de partículas por $\mathrm{cm}^{3}$.

Figura 2 - Distribuição da concentração pela média de volume de interrogação

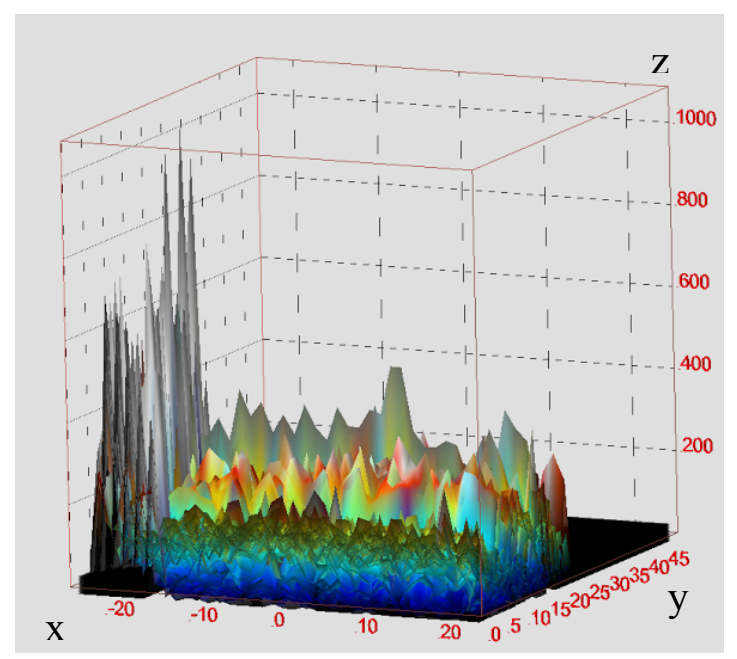

Construiu-se um gráfico com o valor da distribuição média de partículas $\left(1 / \mathrm{cm}^{3}\right)$ ao longo do eixo x (Figura 3) e chegou-se a conclusão que a concentração é aproximadamente constante, característica de um regime em fase diluída. Próximo das bordas seu valor costuma aumentar, pois nesta região se encontra uma fonte de erro devido seções da imagem próximas a paredes do tubo.

Ainda sim é possível notar uma pequena elevação no centro do riser em relação a extremidade direita da Figura 3, pois este é o local de tendência de fluxo das partículas

Figura 3 - Distribuição média da densidade de partículas por $\mathrm{cm}^{3}$ ao longo do eixo horizontal.

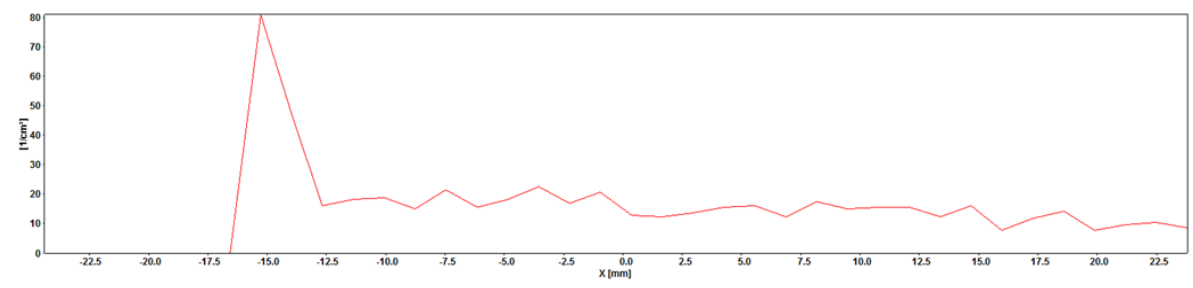

Prosseguindo, os vetores velocidade instantâneos para as sequências de imagem foram obtidos e com eles, calculou-se os vetores de velocidade média como pode ser observado na Figura 4.

Figura 4 - Vetores de velocidade média das partículas em análise 

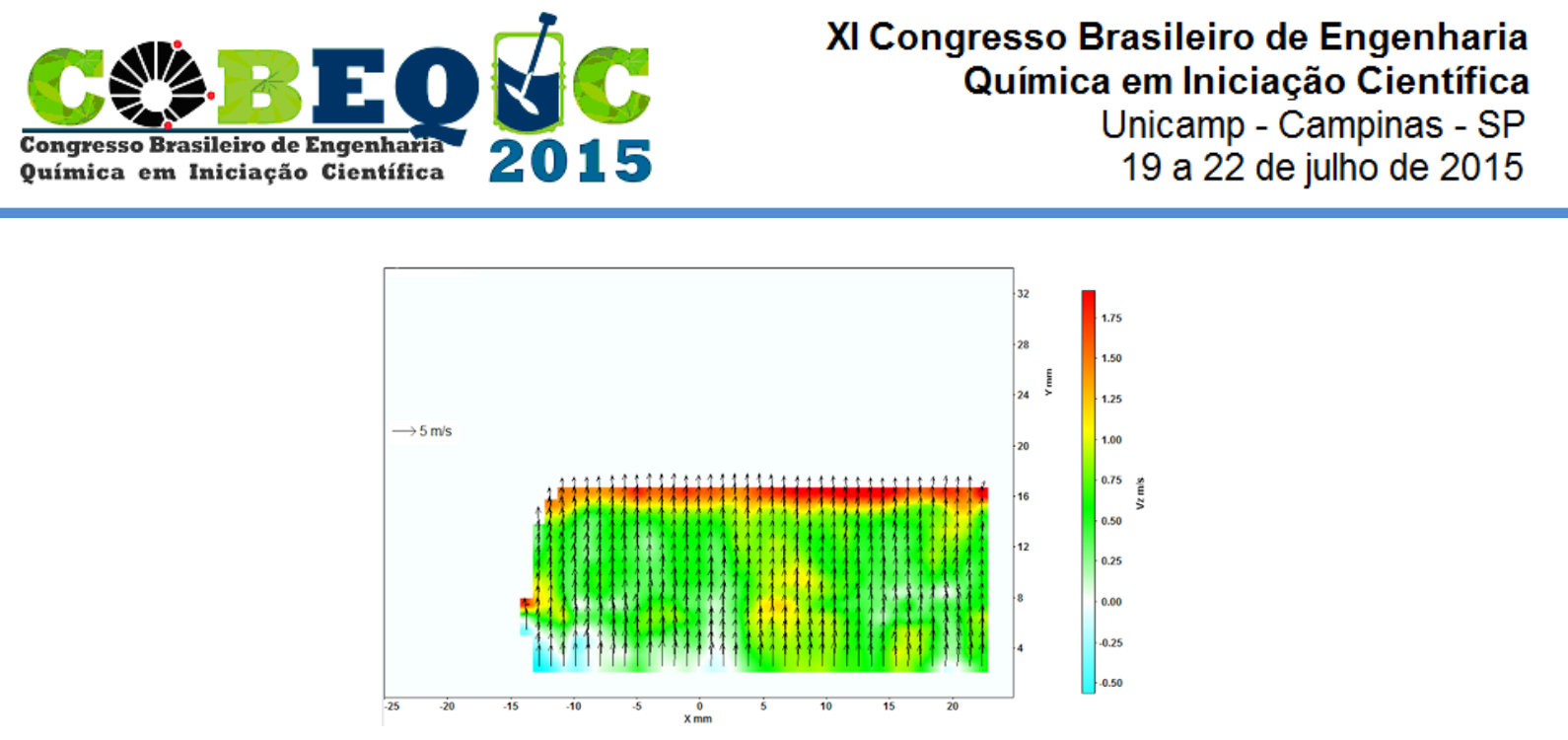

Como é possível observar, os vetores velocidade resultaram numa velocidade baixa próxima a $0 \mathrm{~m} / \mathrm{s}$ para a direção $\mathrm{z}$ e mostrou um deslocamento positivo das partículas para $\mathrm{o}$ sentido positivo no eixo vertical. Isso era esperado, pois fluxo sólido-particulado é nesta direção e sentido. Além disso, por apresentar uma concentração relativamente pequena na região analisada, não ocorre muita interação entre partículas reduzindo a velocidade em direções que não sejam iguais ao do fluxo gás-sólido.

\section{REFERÊNCIAS}

CASTILHO, G. J. Análise de Caos em Leito Fluidizado Circulante. 2011. 183 f. Tese de Doutorado - Faculdade de Engenharia Química, Universidade Estadual de Campinas, Campinas. 2011.

CASTILHO, G. J. Determinação Experimental das Distribuições Radial e Axial de Concentração de Sólidos em uma Seção Riser Utilizando Sonda de Fibras Ópticas. 2007. 119 f. Dissertação de mestrado - Faculdade de Engenharia Química, Universidade Estadual de Campinas, Campinas. 2007.

ADRIAN, R. J. (1991), Particle-imaging techniques for experimental fluid mechanics, Annu. Rev. Fluid Mech., Vol. 23, 261-304.

AMARAL, R. L., CASTILHO, G. J., CREMASCO, M. A. (2012), Identificação da resolução de interrogação na velocimetria por imagem de partícula do tipo tomográfica na investigação do campo de velocidade da fase particulada na seção de um riser. Anais do XiX Congresso Brasileiro de Engenharia Química, Búzios - RJ.

AMARAL, R. L. Efeito da autocalibração volumétrica para PIV tomográfica no campo de velocidade em uma seção de um riser de um leito fluidizado circulante. 2013. $173 \mathrm{f}$. Dissertação (Mestrado em Engenharia Química) - Faculdade de Engenharia Química, Universidade Estadual de Campinas, Campinas. 2013.

LAVISION. Product-Manual: FlowMaster Tomographic PIV. Göttingen. 2010.

WIENEKE, B. Volume self-calibration for 3D particle image velocimetry. Experiments in fluids, v. 45, n. 4, p. 549-556, 2008.

PRASAD, A. Particle image velocimetry - Review article. Current Science, v. 79, p. 51-60, 2000. 